\title{
Leader-Following Consensus of Linear Multiagent Systems with State Observer under Switching Topologies
}

\author{
Lixin Gao, ${ }^{1}$ Xinjian Zhu, ${ }^{2}$ Wenhai Chen, ${ }^{1}$ and Hui Zhang ${ }^{2,3}$ \\ ${ }^{1}$ Institute of Intelligent Systems and Decision, Wenzhou University, Zhejiang 325035, China \\ ${ }^{2}$ Department of Control Science and Engineering, Zhejiang University, Hangzhou 310027, China \\ ${ }^{3}$ State Key Laboratory of Industrial Control Technology, Institute of Industrial Process Control, Zhejiang University, \\ Hangzhou 310027, China
}

Correspondence should be addressed to Hui Zhang; zhanghui@iipc.zju.edu.cn

Received 2 January 2013; Accepted 24 January 2013

Academic Editor: Yang Tang

Copyright (C) 2013 Lixin Gao et al. This is an open access article distributed under the Creative Commons Attribution License, which permits unrestricted use, distribution, and reproduction in any medium, provided the original work is properly cited.

The leader-following consensus problem of higher order multiagent systems is considered. The dynamics of each agent are given in general form of linear system, and the communication topology among the agents is assumed to be directed and switching. To track the leader, two kinds of distributed observer-based consensus protocols are proposed for each following agent, whose distributed observers are used to estimate the leader's state and tracking error based on the relative outputs of the neighboring agents, respectively. Some sufficient consensus conditions are established by using parameter-dependent Lyapunov function method under a class of directed interaction topologies. As special cases, the consensus conditions for balanced and undirected interconnection topology cases can be obtained directly. The protocol design technique is based on algebraic graph theory, Riccati equation, and Sylvester equation. Finally, a simulation example is given to illustrate our obtained result.

\section{Introduction}

In recent years, the coordination control of the multi-agent systems has attracted a great number of researchers. The applications of the multi-agent systems include formation control, flocking, unmanned air vehicles, rendezvous, and distributed computations $[1,2]$. The consensus problem has become a hot topic in the fields of coordination control for multi-agent systems. The main idea of multi-agent consensus is to design the distributed control protocol that enables a group of agents to reach an agreement on certain quantities.

The dynamics model of individual agent and the interacting topology of multi-agent systems are two key factors to achieve consensus. Usually, the stochastic matrix analysis method is used to solve the consensus problems with firstorder agent dynamics under the switching interacting topology [3-6]. In [5], we have pointed out that the first-order continuous-time consensus problem investigated by $[7,8]$ can also be analyzed via the stochastic matrix method.

In many applications, the dynamics of agents are usually modeled by double integrator dynamics (second order).
Although some authors tried to solve the second-order discrete-time consensus problem by using stochastic matrixbased method [9], as [10] has pointed out, the stochastic analysis method may not be applied directly to multi-agent systems with second-order dynamics. From this point, it is nontrivial from first-order consensus to the second-order consensus, and the Lyapunov-based approach is often chosen to solve second-order consensus problem [10-13]. A general framework was introduced by [14] to analyze the consensus problems of multi-agent systems in high-dimensional state space. Compared with the switching interacting topology, it is relatively easy to handle fixed topology by using the eigenvalue decomposition approach [15]. For the switching interacting topology case, the common Lyapunov function (CLF) method is a good substituted way to analyze consensus of multi-agent systems $[10,12,16,17]$. Some other relevant research topics have also been addressed, such as consensus filtering [18], synchronization [19, 20], swarm stability [21, 22], neural network [23], time-delay [24], finite-time consensus [25], and communication constraint [26]. 
The leader-following configuration can be found in many biological systems $[27,28]$, which is very useful to design the multi-agent systems. leader-following consensus problems under jointly connected interacting topology were considered in [3]. In [29], the authors probed the controllability of a Leader-follower dynamic network. To track the active leader, the neighbor-based consensus protocol for each following agent was investigated by $[10,12,17]$. Leader-following consensus problem for multi-agent systems with general linear dynamics was investigated in [30].

In many practical systems especially in sensor networks and robot networks, some variables, which may lead the system to achieve a prescribed group behavior, cannot be obtained directly. To achieve the control goal, the control protocol often contains an observer to estimate those unmeasurable variables. In [31], the authors addressed the problem of output feedback control for networked control systems with limited communication capacity. In [10], the authors proposed an neighbor-based estimation rule for each first-order follower agent to estimate the active leaders'unmeasurable velocity. [16] assumed that each agent can also obtain its neighbor's estimation value and use it in local control rule directly. To track the active leader [12], proposed distributed observer-based control laws for the second-order follower agents under the assumption that the velocity of the active leader cannot be measured. In [32], Abdessameud and Tayebi studied the observer-based consensus algorithm for the second-order agents to measure velocity under input constraints. To track the accelerated motion leader, [17] proposed a neighbor-based estimation rule to estimate the acceleration of the leader. The distributed observer-based cooperative control for multiple nonholonomic mobile agents was addressed by [33]. A distributed algorithm was proposed for the distributed estimation of a general active leader's unmeasurable state variables in [34], and [24] extended the results of [34] to the case of communication delays among agents. Consensus of high-order linear systems was solved by using dynamic output feedback compensator in [35]. A distributed observer-type consensus protocol to solve consensus problem with general linear or linearized agent dynamics under fixed communication topologies was referred to in [36]. A unified framework was introduced in [37] to address the consensus of multiagent systems and the synchronization of complex networks, which proposed an observer-type consensus protocol using only the relative outputs of the neighboring agents under fixed communication topologies. [38] addressed the linear multi-agent consensus problem with discontinuous observations over a time-invariant undirected communication topology. Till now, the problem of observer-based consensus design has become an important topic in the study of multi-agent networks and is attracting more and more researchers.

Motivated by the above works, we study leader-following consensus problem of multi-agent systems with highdimensional linear coupling dynamics under directed switching topology. The main contribution of this paper is that we propose two kinds of distributed observer-based consensus protocols to solve the leader-following consensus problem. The involved observers are used to estimate the leader's state and tracking error based on the relative outputs of neighboring agents, respectively. To construct the consensus protocols, an algorithm based on Riccati equation and Sylvester equation is proposed to design the protocol parameter matrices. By applying the proposed consensus protocols, we prove that the multi-agent system achieves consensus under any directed fixed topology. However, it becomes a challenging problem, when the interaction topology is time varying. By constructing a parameterdependent common Lyapunov function, we prove that the multi-agent system achieves consensus under a class of directed interaction topologies. Obviously, the consensus conditions for some special cases such as balanced and undirected interconnection topology cases can be obtained directly.

The rest of the paper is organized as follows. In Section 2, the formulation of the consensus problem is given with the help of graph theory. Then in Section 3, the distributed consensus protocol based on distributed observer to estimate leader's state is investigated. Similarly, the distributed consensus protocol based on distributed observer to estimate tracking error is considered in Section 4. Following that, Section 5 provides a simulation example, and finally, the concluding remarks are given in Section 6.

\section{Preliminaries and Problem Formulation}

2.1. Problem Formulation. Consider a multi-agent system consisting of $n$ following agents and a leader. The dynamics of each following agent are modeled by the following linear system:

$$
\begin{gathered}
\dot{x}_{i}=A x_{i}+B u_{i}, \quad i=1, \ldots, n, \\
y_{i}=C x_{i},
\end{gathered}
$$

where $x_{i} \in R^{m}$ is the agent is state, $u_{i} \in R^{p}$ is the agent $i$ 's control input, and $y_{i} \in R^{q}$ is the agent $i$ 's measured output. $A, B$, and $C$ are constant matrices with appropriate dimensions.

The leader, labeled as $i=0$, has linear dynamics as

$$
\begin{gathered}
\dot{x}_{0}=A x_{0}+B u_{0}(t), \\
y_{0}=C x_{0},
\end{gathered}
$$

where $x_{0} \in R^{m}$ is the leader's state and $y_{0} \in R^{q}$ is the leader's measured output; the input $u_{0}(t)$ can be regarded as the common policy which is known by all following agents.

Remark 1. This leader-following consensus problem has been investigated by [30], and the special second-order leaderfollowing consensus problem was studied by [11, 12]. [30] assumed that every following agent can obtain the state variables of its neighbors directly. Here, we assume that every following agent can only obtain the measured output of its neighbors directly. As [30] has pointed out, the system matrices for all the agents and the leader were taken to be identical because of their practical background such as group of birds and school of fishes. 
The following assumption is used throughout the paper.

Assumption 1. $(A, B)$ is stabilizable and $(A, C)$ is detectable.

The leader-following multi-agent system is said to be achieved consensus, if the state of any following agent satisfies $\lim _{t \rightarrow \infty}\left(x_{i}(t)-x_{0}(t)\right)=0$, for any initial state $x_{i}(0), i=0,1, \ldots, n$. We say that the protocol $u_{i}(k)$ can solve the consensus control problem, if the closed-loop feedback system achieves consensus.

2.2. Notations and Concepts. Let $R^{m \times n}$ and $C^{m \times n}$ be the set of $m \times n$ real matrices and complex matrices, respectively. $I$ is the identity matrix with compatible dimension. $A^{T}$ and $A^{H}$ represent transpose and conjugate transpose of matrix $A \in C^{m \times n}$, respectively. $\mathbf{1}_{n}=[1, \ldots, 1]^{T} \in R^{n} . \mathscr{I}$ denotes the set $\{1,2, \ldots, n\}$. For symmetric matrices $A$ and $B, A>(\geq) B$ means that $A-B$ is positive (semi)definite. $\|\cdot\|$ denotes Euclidean norm. $\otimes$ denotes the Kronecker product, which satisfies $(1)(A \otimes B)(C \otimes D)=(A C) \otimes(B D)$; (2) if $A \geq 0$ and $B \geq 0$, then $A \otimes B \geq 0$.

To model interconnection topology, some preliminary knowledge of graph theory is introduced. More details are available in [39]. A weighted digraph is denoted by $\mathscr{G}=$ $\{\mathscr{V}, \varepsilon, A\}$, where $\mathscr{V}=\left\{v_{1}, v_{2}, \ldots, v_{n}\right\}$ is the set of vertices, $\varepsilon \subset \mathscr{V} \times \mathscr{V}$ is the set of edges, and a weighted adjacency matrix $A=\left[a_{i j}\right]$ has nonnegative adjacency elements $a_{i j}$ and $a_{i i}=0$. The set of all neighbor nodes of node $v_{i}$ is defined by $\mathcal{N}_{i}=\{j \mid$ $\left.\left(v_{i}, v_{j}\right) \in \varepsilon\right\}$. The degree matrix $D=\left\{d_{1}, d_{2}, \ldots, d_{n}\right\} \in \mathscr{R}^{n \times n}$ of digraph $\mathscr{G}$ is a diagonal matrix with diagonal elements $d_{i}=\sum_{j \in \mathcal{N}_{i}} a_{i j}$. Then the Laplacian matrix of $\mathscr{G}$ is defined as $L=D-A \in R^{n \times n}$.

The in-degree and out-degree of node $v_{i}$ are denoted as $d_{\text {in }}(i)=\sum_{j=1}^{n} a_{j i}$ and $d_{\text {out }}(i)=\sum_{j=1}^{n} a_{i j}$, respectively. A weighted digraph $\mathscr{G}$ is said to be balanced if and only if $d_{\text {in }}(i)=d_{\text {out }}(i)$, for $i=1,2, \ldots, n$. Moreover, a weighted graph $\mathscr{G}$ is balanced if and only if $\mathbf{1}^{T} L=0$, where $\mathbf{1}=(1,1, \ldots, 1)^{T} \in$ $R^{n}$. (see [7]). Certainly, any undirected weighted graph is balanced.

If there is a directed path from node $v_{i}$ to node $v_{j}$, then $v_{j}$ is said to be reachable from $v_{i}$. Node $v_{i}$ is said to be globally reachable if there is a directed path from every other node to node $v_{i}$ in digraph $\mathscr{G}$. A directed graph $\mathscr{G}$ has a globally reachable node if and only if there exists a directed spanning tree in $\mathscr{G}$ (see [4]).

In what follows, we use digraph $\widehat{\mathscr{G}}$ of order $n+1$ to model information topology relation of the multi-agent system that consisted of $n$ agents (labeled as $v_{i}, i=1,2, \ldots, n$ ) and one leader (labeled as $v_{0}$ ) and directed graph $\mathscr{G}$ to model the topology relation of these $n$ followers. In fact, $\widehat{\mathscr{G}}$ contains graph $\mathscr{G}$, and $v_{0}$ with the directed edges from some agents to the leader describes the topology relation among all agents. To describe the variable interconnection topology, the set of all possible topology digraphs is denoted as $S=$ $\left\{\widehat{\mathscr{G}}_{1}, \widehat{\mathscr{G}}_{2}, \ldots, \widehat{\mathscr{G}}_{N}\right\}$ with index set $\mathscr{P}=\{1,2, \ldots, N\}$. The switching signal $\sigma:[0, \infty) \rightarrow \mathscr{P}$ is used to express the index of topology digraph. Let $0=t_{1}, t_{2}, t_{3}, \ldots$ be an infinite time sequence at which the interconnection graph of the considered multi-agent system switches. Therefore, $\mathcal{N}_{i}(t)$ and the connection weights $a_{i j}(t), b_{i}(t)(i, j=1, \ldots, n)$ are time invariant in any interval $\left[t_{i}, t_{i+1}\right)$. Assume that there is a constant $\tau_{0}>0$, often called dwell time, with $t_{i+1}-t_{i} \geq \tau_{0}$, for all $i=1,2,3, \ldots$.

For simplicity, the weights $a_{i j}(t)$ and $b_{i}(t)$ are chosen as follows in our problem:

$a_{i j}(t)= \begin{cases}\alpha_{i j} & \text { if agent } i \text { is connected to agent } j \text { at time } t \\ 0 & \text { otherwise, }\end{cases}$

$b_{i}(t)$

$= \begin{cases}\beta_{i} & \text { if agent } i \text { is connected to the leader at time } t \\ 0 & \text { otherwise }\end{cases}$

where $\alpha_{i j}>0(i, j=1, \ldots, n)$ is the connection weight constant between agent $i$ and agent $j$, and $\beta_{i}>0(i=1, \ldots, n)$ is the connection weight constant between agent $i$ and the leader.

Let $L_{\sigma(t)}$ be the Laplacian matrix of the interaction graph $G_{\sigma(t)} . B_{\sigma(t)}$ is an $n \times n$ diagonal matrix whose $i$ th diagonal element is $b_{i}(t)$ at time $t$. For convenience, let $H_{\sigma(t)}=L_{\sigma(t)}+$ $B_{\sigma(t)}$.

2.3. Preliminary Results. Before establishing our main results, some preliminary results are introduced, which will be used later.

Note that matrix $H=L+B$ plays a key role in the convergence analysis of the system. A matrix is said to be positive stable if all its eigenvalues have positive real parts. The following lemma, which is found in [11], shows a relationship between $H$ and the connectedness of digraph $\widehat{\mathscr{G}}$.

Lemma 2. Matrix $H=L+B$ is positive stable if and only if node 0 is globally reachable in $\widehat{\mathscr{G}}$.

The next two lemmas are well known, whose different versions can be found in many books.

Lemma 3 (see [40]). Let $S$ be a symmetric matrix partitioned as $S=\left[\begin{array}{ll}S_{11} & S_{12} \\ S_{21} & S_{22}\end{array}\right]$, where $S_{11} \in R^{r \times r}, S_{12} \in R^{r \times(n-r)}$, and $S_{22} \in$ $R^{(n-r) \times(n-r)}$. Then $S<0$ if and only if

$$
S_{11}<0, \quad S_{22}-S_{21} S_{11}^{-1} S_{12}<0
$$

or equivalently

$$
S_{22}<0, \quad S_{11}-S_{12} S_{22}^{-1} S_{21}<0 .
$$

Lemma 4 (see [41]). If $(A, C)$ is detectable and $Q$ is a symmetric positive definite matrix, then there is a unique positive definite matrix $P$ to satisfy the Riccati equation

$$
P A^{T}+A P-P C^{T} C P+Q=0 .
$$

Furthermore, the real parts of all the eigenvalues of $A^{T}-$ $C^{T} C P$ are negative. 


\section{Distributed State Observer to Estimate the Leader's State}

To solve the leader-following consensus problem, the relative measurement is involved. Let $z_{i}$ be the relative output error of agent $i$ with its neighbor agent as follows:

$$
z_{i}=\sum_{j \in \mathcal{N}_{i}} a_{i j}(t)\left(\bar{y}_{i}-\bar{y}_{j}\right)+\beta_{i}(t)\left(\bar{y}_{i}-y_{0}\right),
$$

where $\bar{y}_{i}=C \bar{x}_{i}$ is the output of the estimator, the connection weights $a_{i j}(t)$ and $b_{i}(t)$ are defined as (3) and (4), respectively. Then, we propose a distributed control protocol for agent $i$ as follows, which consists of a distributed estimation law and a feedback control law:

(i) distributed estimation law for agent $i$ :

$$
\dot{\bar{x}}_{i}=A \bar{x}_{i}-\kappa L z_{i}+B u_{0}(t), \quad i=1, \ldots, n,
$$

where $\bar{x}_{i} \in R^{m}$ is the protocol state, $\kappa$ is the coupling strength, and $L \in R^{m \times q}$ is a given gain matrix;

(ii) feedback control law for agent $i$ :

$$
u_{i}=u_{0}(t)-K\left(x_{i}-\bar{x}_{i}\right) \quad i=1,2, \ldots, n,
$$

where $K$ is a given feedback gain matrix.

In fact, estimation law (9) plays the role of state observer for agent $i$ to estimate the leader's state variables. From (9), each agent relies only on the locally available information at every moment. A following agent cannot "observe" or "estimate" the leader directly based on the measured information of the leader if it is not connected to the leader. Thus, it has to collect the information of the leader in a distributed way from its neighbor agents.

Our objective is to design $\kappa, L, K$ to make the leaderfollowing multi-agent system achieve consensus. To this end, the following algorithm is presented to construct the gain matrix $L$ and the feedback matrix $K$ in state estimation law (9) and control law (10).

Algorithm 5. Given that $(A, B, C)$ is stabilizable and detectable, the gain matrix $L$ and feedback matrix $K$ are constructed as follows:

(1) for a given positive definite matrix $Q$, solve the following Riccati equation

$$
A P+P A^{T}-P C^{T} C P+Q=0
$$

to obtain the unique positive definite matrix $P$. Then, the gain matrix $L$ is chosen by $L=P C^{T}$;

(2) choose $K$ such that $A-B K$ is stable.

Remark 6. One method to construct the feedback matrix $K$ is introduced as follows:
(2.1) select a stable $n \times n$ matrix $F$ with a set of desired eigenvalues that contains no eigenvalues in common with those of $A$;

(2.2) select $\bar{K}$ randomly such that $(F, \bar{K})$ is observable;

(2.3) solve Sylvester equation

$$
A T-T F=B \bar{K}
$$

to get a nonsingular solution $T$. If $T$ is singular, select another $\bar{K}$, until $T$ is nonsingular;

(2.4) compute $T^{-1}$ and take $K=\bar{K} T^{-1}$. From (2.1)-(2.4) of Algorithm 5, it's easy to get

$$
\begin{aligned}
A-B K & =A-B \bar{K} T^{-1} \\
& =(A T-B \bar{K}) T^{-1}=T F T^{-1}
\end{aligned}
$$

which means that $A-B K$ is stable. The above method, to construct feedback matrix $K$, can be found in [42]. Of course, there are several other methods to, construct matrix $K$ if $(A, B)$ is stabilizable. From Lemma 4, the Riccati equation (11) is soluble if $(A, C)$ is detectable. Thus, a sufficient condition for Algorithm 5 to construct protocols (9) and (10) successfully is that $(A, B)$ is stabilizable and $(A, C)$ is detectable.

Denote $\bar{e}_{i}=\bar{x}_{i}-x_{0}$ and $\bar{e}=\left(\bar{e}_{1}^{T}, \bar{e}_{2}^{T}, \ldots, \bar{e}_{n}^{T}\right)^{T}$. Then, after manipulations with combining (1), (2), and (8), we have

$$
\begin{aligned}
\dot{\bar{e}}_{i}= & A \bar{e}_{i}-\kappa L \\
& \times\left[\sum_{j \in \mathcal{N}_{i}} a_{i j}(t) C\left(\bar{e}_{i}-\bar{e}_{j}\right)+\beta_{i}(t) C \bar{e}_{i}\right],
\end{aligned}
$$

which can be written in stack vector form:

$$
\dot{\bar{e}}=\left[(I \otimes A)-\left(L_{\sigma(t)}+B_{\sigma(t)}\right) \otimes(\kappa L C)\right] \bar{e} .
$$

Similarly, taking $e_{i}=x_{i}-x_{0}$ and $e=\left(e_{1}^{T}, e_{2}^{T}, \ldots, e_{N}^{T}\right)^{T}$, we have

$$
\begin{aligned}
\dot{e}_{i} & =\dot{x}_{i}-\dot{x}_{0} \\
& =A\left(x_{i}-x_{0}\right)+B K\left(\bar{x}_{i}-x_{0}+x_{0}-x_{i}\right) \\
& =(A-B K) e_{i}+B K \bar{e}_{i}
\end{aligned}
$$

or equivalently

$$
\dot{e}=I \otimes(A-B K) e+I \otimes B K \bar{e} .
$$


From (15) and (17), the error dynamics system will be expressed in a compact form as follows:

$$
\dot{\varepsilon}(t)=F_{\sigma} \varepsilon(t),
$$

where

$$
\begin{aligned}
& \varepsilon(t)=\left(\begin{array}{l}
e \\
\bar{e}
\end{array}\right), \\
& F_{\sigma}=\left(\begin{array}{cc}
I \otimes(A-B K) & I \otimes(B K) \\
0 & I \otimes A-H_{\sigma} \otimes(\kappa L C)
\end{array}\right) .
\end{aligned}
$$

Obviously, the multi-agent system achieves consensus if $\lim _{t \rightarrow \infty} \varepsilon(t)=0$. Thus, the leader-following consensus problem of multi-agent system is transformed into the stability problem of error dynamic system (18).

3.1. Fixed Interconnection Topology Case. In this subsection, the leader-following consensus problem under fixed interconnection topology is investigated. In this case, the error system can be rewritten as follows by dropping the subscript $\sigma(t)$

$$
\begin{aligned}
& \dot{\varepsilon}(t) \\
& \quad=\left(\begin{array}{cc}
I \otimes(A-B K) & I \otimes(B K) \\
0 & I \otimes A-H \otimes(\kappa L C)
\end{array}\right) \varepsilon(t) \triangleq F \varepsilon(t) .
\end{aligned}
$$

Now, we present the following result for the fixed interconnection topology case.

Theorem 7. Suppose that the interconnection topology $\widehat{\mathscr{G}}$ is fixed with globally reachable node $v_{0}$ and the matrices $L, K$ used in control protocol are constructed by Algorithm 5. Take the coupling strength $\kappa$ satisfying

$$
\kappa \geq \frac{1}{2 \min _{i \in \mathscr{I}} \operatorname{Re}\left(\lambda_{i}\right)},
$$

where $\lambda_{i}$ is ith eigenvalue of $H$. Then, the distributed control protocols (9) and (10) can guarantee that all following agents track the leader from any initial condition.

Proof. By applying Schur orthogonal decomposition to matrix $H$, there exists a unitary matrix $U$ such that

$$
U H U^{H}=\left(\begin{array}{cccc}
\lambda_{1} & * & \cdots & * \\
0 & \lambda_{2} & \cdots & * \\
\vdots & \vdots & \vdots & \vdots \\
0 & 0 & \cdots & \lambda_{n}
\end{array}\right) \triangleq \Delta .
$$

From (22), we have

$$
\begin{gathered}
\left(\begin{array}{cc}
I_{n} \otimes I_{m} & \\
& U \otimes I_{m}
\end{array}\right) F\left(\begin{array}{cc}
I_{n} \otimes I_{m} & \\
& U \otimes I_{m}
\end{array}\right)^{H} \\
\quad=\left(\begin{array}{cc}
I \otimes(A-B K) & I \otimes(B K) \\
0 & I \otimes A-\Delta \otimes(\kappa L C)
\end{array}\right) .
\end{gathered}
$$

Then, it is easy to see that $F$ is stable if and only if $A-\lambda_{i} \kappa L C$ is stable for any $i=1,2, \ldots, n$. From the fact that positive definite matrix $P$ is a unique solution of (11) and $L=P C^{T}$, we can obtain

$$
\begin{aligned}
& P\left(A-\lambda_{i} \kappa L C\right)^{H}+\left(A-\lambda_{i} \kappa L C\right) P \\
& \quad=-Q+P C^{T} C P-2 \operatorname{Re}\left(\lambda_{i}\right) \kappa P C^{T} C P \leq-Q,
\end{aligned}
$$

which implies that $A-\lambda_{i} \kappa L C$ is stable. Thus, we have $\lim _{t \rightarrow \infty} \varepsilon(t)=0$. The proof is now completed.

Remark 8. Since the interconnection topology $\widehat{\mathscr{G}}$ is fixed with globally reachable node $v_{0}$, matrix $H$ is positive stable according to Lemma 2. Thus, $\min _{i \in \mathscr{I}} \operatorname{Re}\left(\lambda_{i}\right)$ is well defined and greater than zero. On the other hand, if the node $v_{0}$ is not globally reachable, at least a node must exist from which there is no directed path to node $v_{0}$ in graph $\widehat{\mathscr{G}}$. This means that some following agents always do not get the state information of the leader directly or indirectly. Certainly, the multi-agent system may not achieve consensus for any given initial condition in this case. Thus, the condition that node 0 is globally reachable in $\widehat{\mathscr{G}}$ is also necessary to achieve consensus under fixed interconnection topology.

3.2. Switching Topology Case. Now, we discuss the convergence analysis of system (18) under switching interconnection topology. For convenience, a class of interconnection topology graphs is defined by the following:

$$
\begin{gathered}
\Gamma=\left\{\widehat{\mathscr{G}} \mid v_{0} \text { is a globally reachable node in graph } \widehat{\mathscr{G}}\right. \\
\text { and } \left.H^{T}(\widehat{\mathscr{G}})+H(\widehat{\mathscr{G}}) \text { is positive definite }\right\} .
\end{gathered}
$$

Therefore, define

$$
\bar{\lambda}:=\min _{\widehat{\mathscr{G}} \in \operatorname{S} \cap \Gamma}\left\{\lambda\left(H^{T}(\widehat{\mathscr{G}})+H(\widehat{\mathscr{G}})\right)\right\} .
$$

Noticing that the set $S \cap \Gamma$ is a finite set and $H^{T}(\widehat{\mathscr{G}})+H(\widehat{\mathscr{G}})$ is a positive definite, we know that $\bar{\lambda}$ is well defined, which is positive and depends directly on the constants $a_{i j}$ and $\beta_{i}$ $(i, j=1,2, \ldots, n)$ given in (3) and (4).

The convergence analysis result for multi-agent consensus under switching interconnection topology by using the parameter-dependent Lyapunov function method is given in the following theorem.

Theorem 9. Assume that $\widehat{\mathscr{G}}_{\sigma(t)} \in S \subset \Gamma$ in any interval $\left[t_{j}, t_{j+1}\right)$ and the matrices $L, K$ used in control protocol are constructed by Algorithm 5. Take the coupling strength $\kappa$ satisfying

$$
\kappa \geq \frac{1}{\bar{\lambda}} .
$$

The distributed control protocols (9) and (10) can guarantee that all following agents track the leader from any initial condition. 
Proof. To prove the theorem, we first consider the error dynamics in each interval. In any interval $\left[t_{i}, t_{i+1}\right)$, the topology graph is fixed and the system matrices are time invariant with some fixed $\sigma(t)=p \in \mathscr{P}$. Let $U_{p}$ be an orthogonal transformation such that $U_{p}\left(H_{p}^{T}+H_{p}\right) U_{p}^{T}$ is a diagonal matrix $\Lambda_{p}=\operatorname{diag}\left\{\lambda_{1 p}, \lambda_{2 p}, \ldots, \lambda_{n p}\right\}$, where $\lambda_{i p}$ is the $i$ th eigenvalue of matrix $H_{p}^{T}+H_{p}$.

According to Algorithm 5 and condition (27), we can know that the unique solution $P>0$ of Riccati equation satisfies

$$
\begin{aligned}
P(A & \left.-\frac{1}{2} \lambda_{i p} \kappa L C\right)^{T}+\left(A-\frac{1}{2} \lambda_{i p} \kappa L C\right) P \\
& =-Q+P C^{T} C P-\lambda_{i p} \kappa P C^{T} C P \\
& \leq-Q
\end{aligned}
$$

which implies the following inequality:

$$
\begin{aligned}
(I \otimes P) & \left(I \otimes A-\frac{1}{2} \Lambda_{p} \otimes(\kappa L C)\right)^{T} \\
& +\left(I \otimes A-\frac{1}{2} \Lambda_{p} \otimes(\kappa L C)\right)(I \otimes P) \\
\leq & -I \otimes Q<0 .
\end{aligned}
$$

By pre- and postmultiplying the above inequality (29) with $U_{p} \otimes I$ and its transpose, respectively, we have

$$
\begin{aligned}
(I \otimes P) & \left(I \otimes A-\frac{1}{2}\left(H_{p}^{T}+H_{p}\right) \otimes(\kappa L C)\right)^{T} \\
& +\left(I \otimes A-\frac{1}{2}\left(H_{p}^{T}+H_{p}\right) \otimes(\kappa L C)\right)(I \otimes P) \\
& \leq-I \otimes Q<0 .
\end{aligned}
$$

Thus, the following inequality holds by noting that $L C P$ is a symmetric matrix:

$$
\begin{aligned}
(I \otimes P) & \left(I \otimes A-H_{p} \otimes(\kappa L C)\right)^{T} \\
& +\left(I \otimes A-H_{p} \otimes(\kappa L C)\right)(I \otimes P) \\
= & (I \otimes P)\left[I \otimes A-\frac{1}{2}\left(H_{p}^{T}+H_{p}\right) \otimes(\kappa L C)\right]^{T} \\
& +\left[I \otimes A-\frac{1}{2}\left(H_{p}^{T}+H_{p}\right) \otimes(\kappa L C)\right](I \otimes P) \\
\leq & -I \otimes Q<0 .
\end{aligned}
$$

Set $P_{1}=P^{-1}$ and $Q_{1}=P_{1} Q P_{1} . P_{1}$ and $Q_{1}$ are both positive definite matrices. From (33), it is not hard to obtain the following inequality:

$$
\begin{aligned}
\left(I \otimes P_{1}\right)\left(I \otimes A-H_{p} \otimes(\kappa L C)\right) \\
\quad+\left(I \otimes A-H_{p} \otimes(\kappa L C)\right)^{T}\left(I \otimes P_{1}\right) \\
\leq-I \otimes Q_{1}<0 .
\end{aligned}
$$

According to step (2) of Algorithm 5, $A-B K$ is stable; that is, there exist positive definite matrices $Q_{2}$ and $P_{2}$ satisfying the Lyapunov equation

$$
P_{2}(A-B K)+(A-B K)^{T} P_{2}=-Q_{2} .
$$

Choose the following parameter-dependent Lyapunov matrix:

$$
\widetilde{P}=\left(\begin{array}{cc}
\frac{1}{\omega} I \otimes P_{2} & 0 \\
0 & I \otimes P_{1}
\end{array}\right),
$$

where $\omega$ is positive parameter. Obviously, $\widetilde{P}$ is positive matrix. Then, consider the following common Lyapunov function for error dynamic system (18):

$$
V(\varepsilon(t))=\varepsilon(t)^{T} \widetilde{P} \varepsilon(t) .
$$

For any interval $\left[t_{i}, t_{i+1}\right)$, the time derivative of this Lyapunov function along the trajectory of system (18) is

$$
\frac{d}{d t} V(\varepsilon)=\varepsilon^{T}\left(F_{\sigma}^{T} \widetilde{P}+\widetilde{P} F_{\sigma}\right) \varepsilon=\varepsilon^{T} Q_{\sigma} \varepsilon
$$

where

$$
\begin{gathered}
Q_{\sigma}=\left(\begin{array}{cc}
\frac{1}{\omega} I \otimes\left(P_{2}(A-B K)+(A-B K)^{T} P_{2}\right) & \frac{1}{\omega} I \otimes\left(P_{2} B K\right) \\
\frac{1}{\omega} I \otimes\left(K^{T} B^{T} P_{2}\right) & Q_{2 \sigma}
\end{array}\right), \\
Q_{2 \sigma}=\left(I \otimes P_{1}\right)\left(I \otimes A-H_{\sigma} \otimes(\kappa L C)\right) \\
+\left(I \otimes A-H_{\sigma} \otimes(\kappa L C)\right)^{T}\left(I \otimes P_{1}\right) .
\end{gathered}
$$

According to (30) and (33), we have

$$
Q_{\sigma} \leq\left(\begin{array}{cc}
-\frac{1}{\omega} I \otimes Q_{2} & \frac{1}{\omega} I \otimes\left(P_{2} B K\right) \\
\frac{1}{\omega} I \otimes\left(K^{T} B^{T} P_{2}\right) & -I \otimes Q_{1}
\end{array}\right):=-\bar{Q} .
$$

Choose $\omega$ satisfying

$$
\omega>\lambda_{\max }\left(Q_{1}^{-1}\left(P_{2} B K\right)^{T} Q_{2}^{-1}\left(P_{2} B K\right)\right),
$$

which implies that

$$
\omega Q_{1}>\left(P_{2} B K\right)^{T} Q_{2}^{-1}\left(P_{2} B K\right) .
$$

According to Lemma 3, we know that matrix $\bar{Q}$ is positive definite while condition (39) is satisfied.

It is well known that Lyapunov function $V(\varepsilon)$ satisfies

$$
\lambda_{\min }(\widetilde{P})\|\varepsilon\|^{2} \leq V(\varepsilon) \leq \lambda_{\max }(\widetilde{P})\|\varepsilon\|^{2} .
$$

Therefore, we have $\|\varepsilon\| \leq \sqrt{V(\varepsilon) / \lambda_{\min }(\widetilde{P})}$. On the other hand, we know that

$$
\min \frac{\varepsilon^{T} \bar{Q} \varepsilon}{\mathcal{E}^{T} \widetilde{P} \varepsilon} \geq \frac{\lambda_{\min }(\bar{Q})}{\lambda_{\max }(\widetilde{P})} .
$$


Let $\beta=\lambda_{\min }(\bar{Q}) / \lambda_{\max }(\widetilde{P})$. Therefore, from (36), we have $(d / d t) V(\varepsilon) \leq-\beta V(\varepsilon)$ or equivalently $V(\varepsilon) \leq V(\varepsilon(0)) e^{-\beta t}$. Thus, $\lim _{t \rightarrow \infty} \varepsilon(t)=0$ is satisfied, which means that the leader-following consensus problem is solved by control law (10) together with state estimation law (9). The proof is now completed.

Remark 10. For the special case that the graph $\mathscr{G}_{\sigma}$ associated with all followers is balanced, matrix $L_{\sigma}+L_{\sigma}^{T}$ is positive semidefinite in this case [7]. Moreover, suppose that $\mathscr{G}_{l}$ is balanced. Then $H_{l}^{T}+H_{l}$ is positive definite if and only if $v_{0}$ is globally reachable node in $\widehat{\mathscr{G}}_{l}$ [11]. Thus, $\Gamma$ is not empty and at least concludes a class of interconnection topology $\widehat{\mathscr{G}}$ whose $\mathscr{G}$ associated with all followers is balanced and $v_{0}$ is globally reachable node in $\widehat{\mathscr{G}}$. The undirected interconnection topology considered in $[10,24,30]$ also belongs to $\Gamma$. Therefore, our established results can be applied to those special cases directly.

\section{Distributed State Observer to Estimate Tracking Error}

In this section, we propose another distributed control protocol for agent $i$, which also consists of a distributed estimation law and a feedback control law. The distributed estimation law plays the role of state observer to estimate tracking error instead of leader's state. The involved relative output error of agent $i$ with its neighbor agent is taken as

$$
\begin{gathered}
\widetilde{z}_{i}=\sum_{j \in \mathcal{N}_{i}} a_{i j}(t)\left[\left(\tilde{y}_{i}-\tilde{y}_{j}\right)-\left(y_{i}-y_{j}\right)\right] \\
+\beta_{i}(t)\left[\tilde{y}_{i}-\left(y_{i}-y_{0}\right)\right],
\end{gathered}
$$

where $\tilde{y}_{i}=C \tilde{x}_{i}$ and the connection weights $a_{i j}(t)$ and $b_{i}(t)$ are taken as (3) and (4), respectively.

Then, another kind of distributed control protocol for agent $i$ is given as follows:

(i) distributed estimation law for agent $i$ to estimate tracking error:

$$
\dot{\tilde{x}}_{i}=(A-B K) \widetilde{x}_{i}-\kappa L \widetilde{z}_{i}, \quad i=1, \ldots, n,
$$

where $\tilde{x}_{i} \in R^{m}$ is the protocol state;

(ii) feedback control law for agent $i$ :

$$
u_{i}=u_{0}(t)-K \tilde{x}_{i} \quad i=1,2, \ldots, n .
$$

Here, the control parameter $\kappa$, gain matrix $L$, and feedback matrix $K$ are defined and selected as the previous section.

Denote $\tilde{e}_{i}=\tilde{x}_{i}-\left(x_{i}-x_{0}\right)$ and $\tilde{e}=\left(\widetilde{e}_{1}^{T}, \tilde{e}_{2}^{T}, \ldots, \tilde{e}_{n}^{T}\right)^{T}$. From (1), (2), (43), and (45), we have

$$
\dot{\tilde{e}}_{i}=A \widetilde{e}_{i}-\kappa L\left[\sum_{j \in N_{i}} a_{i j}(t) C\left(\widetilde{e}_{i}-\widetilde{e}_{j}\right)+\beta_{i}(t) C \widetilde{e}_{i}\right],
$$

which can be written in stack vector form:

$$
\begin{aligned}
\dot{\tilde{e}} & =\left[(I \otimes A)-\left(L_{\sigma(t)}+B_{\sigma(t)}\right) \otimes(L C)\right] \widetilde{e} \\
& =\left[(I \otimes A)-H_{\sigma(t)} \otimes(L C)\right] \widetilde{e} .
\end{aligned}
$$

Similarly, for the tracking error $e_{i}=x_{i}-x_{0}$ and $e=$ $\left(e_{1}^{T}, e_{2}^{T}, \ldots, e_{N}^{T}\right)^{T}$, we can obtain

$$
\begin{aligned}
\dot{e}_{i} & =\dot{x}_{i}-\dot{x}_{0}=A\left(x_{i}-x_{0}\right)+B\left(u_{i}-u_{0}\right) \\
& =A\left(x_{i}-x_{0}\right)-B K \widetilde{x}_{i}=(A-B K) e_{i}-B K \widetilde{e}_{i}
\end{aligned}
$$

or equivalently

$$
\dot{e}=I \otimes(A-B K) e-I \otimes(B K) \tilde{e} .
$$

From (47) and (49), the error dynamics system will be expressed in a compact form as follows:

$$
\dot{\tilde{\varepsilon}}(t)=\widetilde{F}_{\sigma} \widetilde{\varepsilon}(t),
$$

where

$$
\begin{gathered}
\widetilde{\varepsilon}(t)=\left(\begin{array}{l}
e \\
\tilde{e}
\end{array}\right), \\
\widetilde{F}_{\sigma}=\left(\begin{array}{cc}
I \otimes(A-B K) & -I \otimes(B K) \\
0 & I \otimes A-H_{\sigma} \otimes L C
\end{array}\right) .
\end{gathered}
$$

Obviously, the multi-agent system achieves consensus if $\lim _{t \rightarrow \infty} \widetilde{\varepsilon}(t)=0$. Thus, the leader-following consensus problem of multi-agent system discussed in this paper is transformed into the stability problem of error dynamic system (50).

Although we use different control protocol for each agent, we get similar error dynamic system. There is just one difference between error dynamic system (50) and error dynamic system (18). By using similar analysis approach, we can also solve the consensus problem under fixed and switching topology cases. The proofs are omitted because it is quite similar to the proofs of Theorems 7 and 9 , respectively.

Theorem 11. Suppose that the interconnection topology $\widehat{\mathscr{G}}$ is fixed with globally reachable node $v_{0}$ and the matrices $L, K$ used in control protocol are constructed by Algorithm 5. Take the coupling strength $\kappa$ satisfying (21). Then, the distributed control protocols (44) and (45) can guarantee that all following agents track the leader from any initial condition.

Theorem 12. Assume that $\widehat{\mathscr{G}}_{\sigma(t)} \in S \subset \Gamma$ in any interval $\left[t_{j}, t_{j+1}\right)$ and the matrices $L, K$ used in control protocol are constructed by Algorithm 5. Take the coupling strength $\kappa$ satisfying (27). The distributed control protocols (44) and (45) can guarantee that all following agents track the leader from any initial condition.

Remark 13. Of course, the established results of this section can be also applied to the balanced interconnection topology and undirected interconnection topology cases directly. Compared with the system matrix $A$ used in estimation (9), the system matrix $A-B K$ used in estimation law (44) must be stable, and it does not use the agent $i$ 's state $x_{i}$ in feedback control law (45). Thus, the distributed control protocols (44) and (45) may be more accepted in applications. 


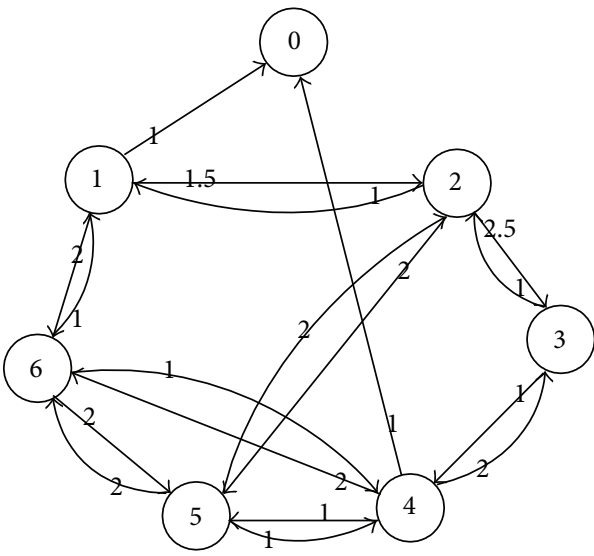

(a)

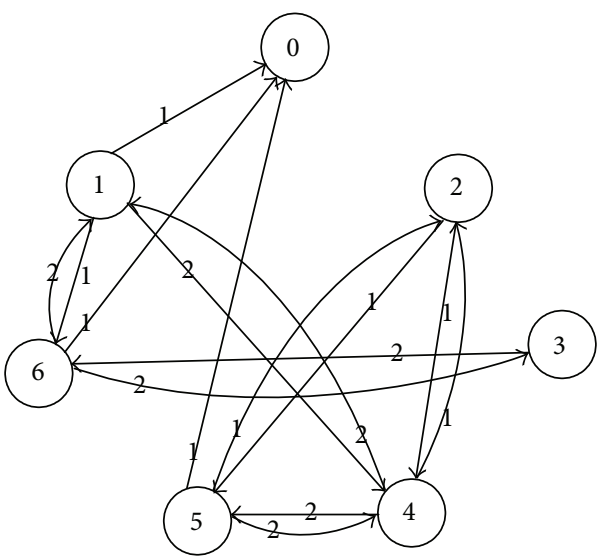

(c)

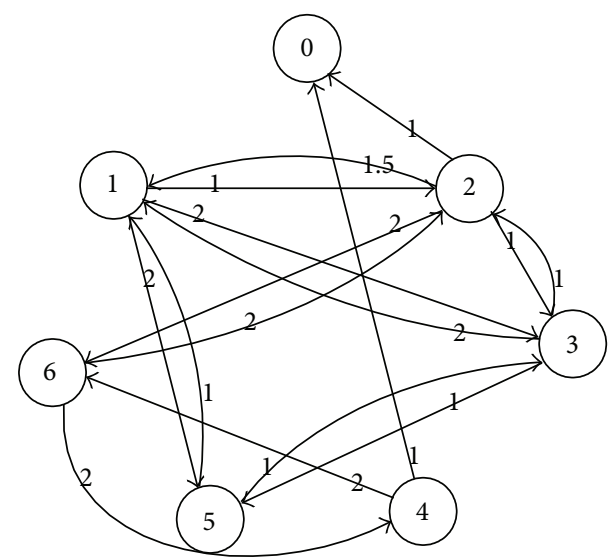

(b)

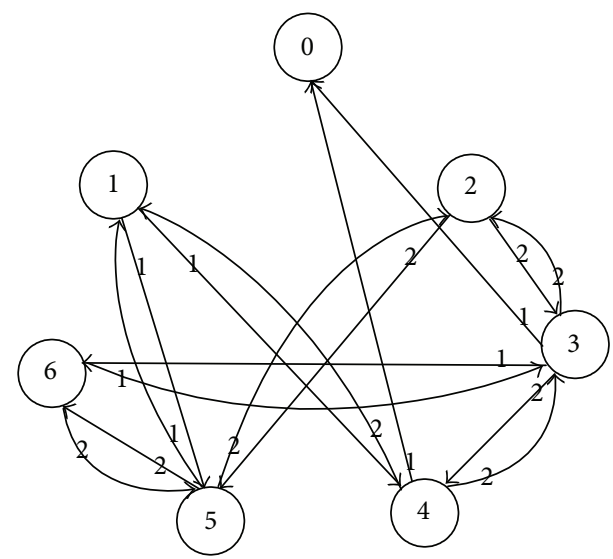

(d)

FIGURE 1: Four interconnection topology graphs.

\section{Simulation Example}

In this section, a numerical simulation is given to illustrate the theoretical results obtained in the previous sections. The multi-agent system is consisted of one leader and six followers. The system matrices of agent dynamics in (1) and (2) are given by

$$
\begin{gathered}
A=\left[\begin{array}{ccc}
-1 & -2 & -3 \\
-2 & -2 & 1 \\
-3 & 1 & 1
\end{array}\right], \\
B=\left[\begin{array}{cc}
1 & 0 \\
1 & -1 \\
2 & 1
\end{array}\right], \\
C=\left[\begin{array}{lll}
1 & 0 & -1 \\
0 & 3 & -2
\end{array}\right] .
\end{gathered}
$$

Without loss of generality, we take $u_{0}(t)=0$ in numerical simulation. The interconnection topologies are arbitrarily switched among three graphs $\widehat{\mathscr{G}}_{i}(i=1,2,3,4)$, which are shown in Figure 1.

The Laplacian matrices $L_{i}(i=1,2,3,4)$ for the four subgraphs $\mathscr{G}_{i}(i=1,2,3,4)$ are

$$
\begin{aligned}
L_{1} & =\left(\begin{array}{cccccc}
3.5 & -1.5 & 0 & 0 & 0 & -2 \\
-1 & 5.5 & -2.5 & 0 & -2 & 0 \\
0 & -1 & 2 & -1 & 0 & 0 \\
0 & 0 & -2 & 5 & -1 & -2 \\
0 & -2 & 0 & -1 & 5 & -2 \\
-1 & 0 & 0 & -1 & -2 & 4
\end{array}\right) \\
L_{2} & =\left(\begin{array}{cccccc}
5 & -1 & -2 & 0 & -2 & 0 \\
-1.5 & 4.5 & -1 & 0 & 0 & -2 \\
-2 & -1 & 4 & 0 & -1 & 0 \\
0 & 0 & 0 & 2 & 0 & -2 \\
-1 & 0 & -1 & 0 & 2 & 0 \\
0 & -2 & 0 & -2 & 0 & 4
\end{array}\right)
\end{aligned}
$$




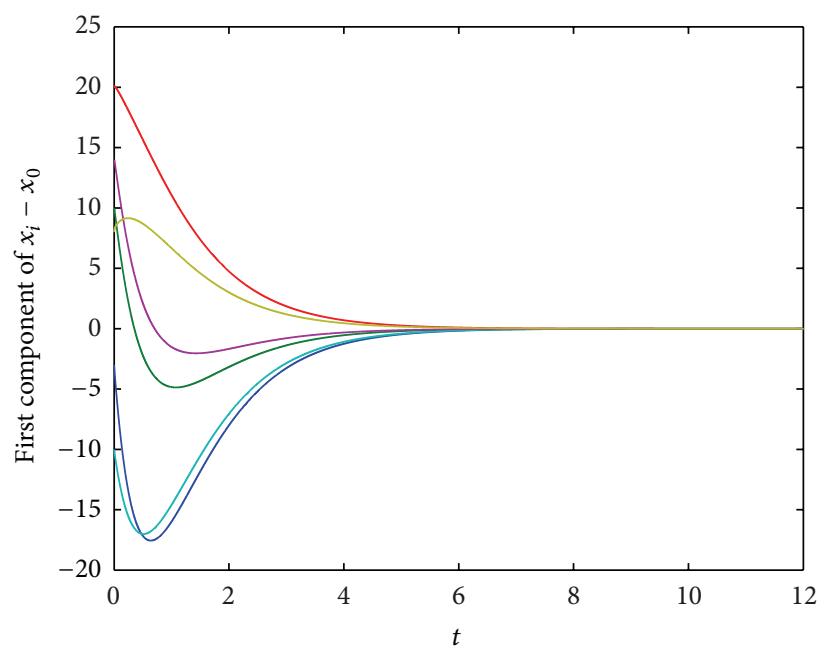

(a)

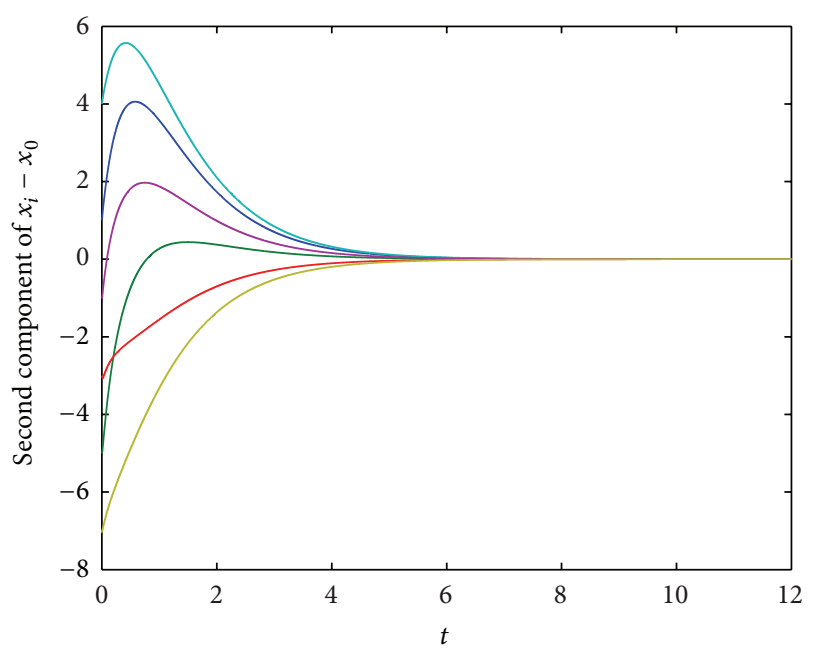

(b)

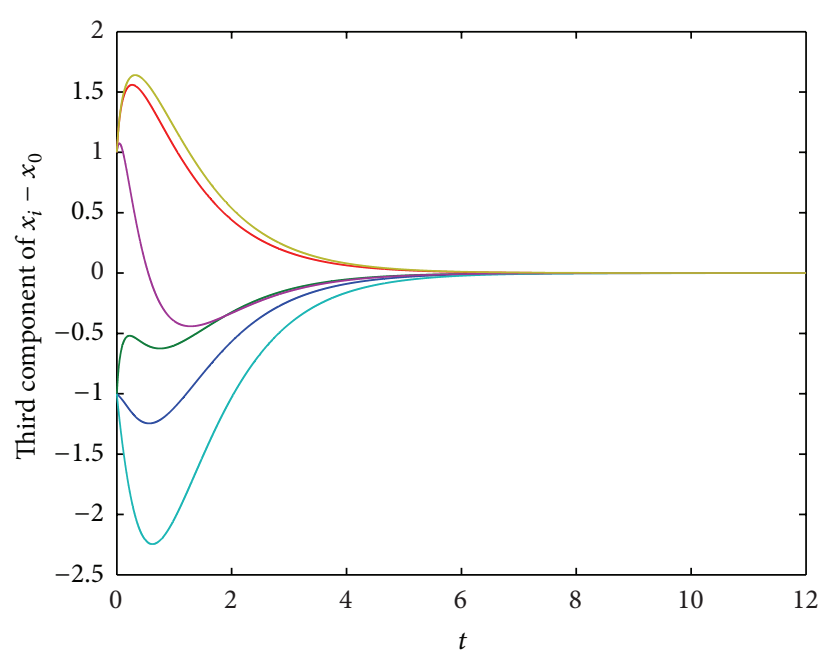

(c)

Figure 2: The error trajectories between the leader and each agent.

$$
\begin{aligned}
L_{3} & =\left(\begin{array}{cccccc}
3 & 0 & 0 & -2 & 0 & -1 \\
0 & 2 & 0 & -1 & -1 & 0 \\
0 & 0 & 2 & 0 & 0 & -2 \\
-2 & -1 & 0 & 5 & -2 & 0 \\
0 & -1 & 0 & -2 & 3 & 0 \\
-2 & 0 & -2 & 0 & 0 & 4
\end{array}\right) \\
L_{4} & =\left(\begin{array}{cccccc}
2 & 0 & 0 & -1 & -1 & 0 \\
0 & 4 & -2 & 0 & -2 & 0 \\
0 & -2 & 5 & -2 & 0 & -1 \\
-2 & 0 & -2 & 4 & 0 & 0 \\
-1 & -2 & 0 & 0 & 5 & -2 \\
0 & 0 & -1 & 0 & -2 & 3
\end{array}\right)
\end{aligned}
$$

and the diagonal matrices for the interconnection relationship between the leader and the followers are

$$
\begin{aligned}
& B_{1}=\operatorname{diag}(1,0,0,1,0,0), \\
& B_{2}=\operatorname{diag}(0,1,0,1,0,0), \\
& B_{3}=\operatorname{diag}(1,0,0,0,1,1), \\
& B_{4}=\operatorname{diag}(0,0,1,1,0,0) .
\end{aligned}
$$

All $H_{i}=L_{i}+B_{i}, i=1,2,3,4$, is not symmetric and satisfies $H_{i}+H_{i}^{T}>0$. Then, we know that $\bar{\lambda}=0.095$. Take $\kappa=1>1 / \bar{\lambda}$. 


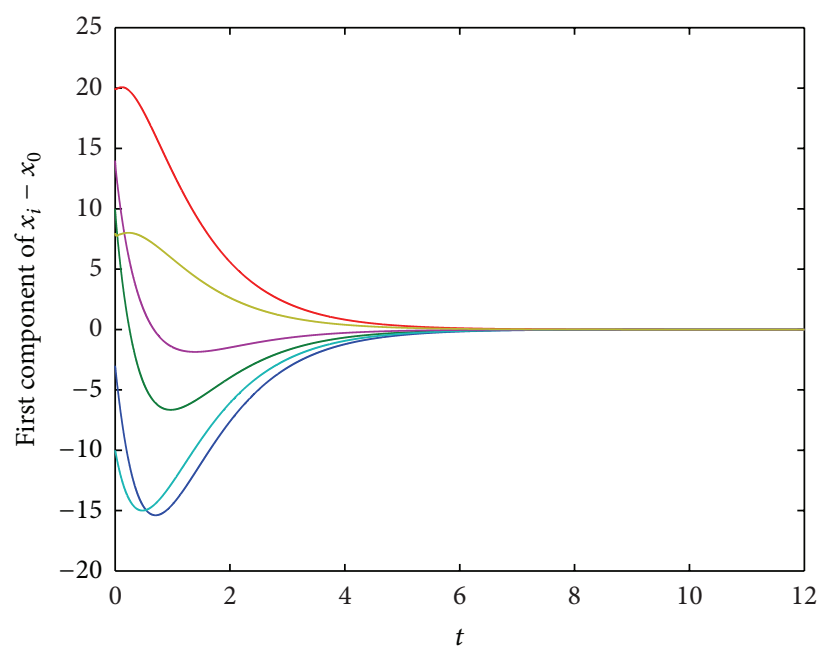

(a)

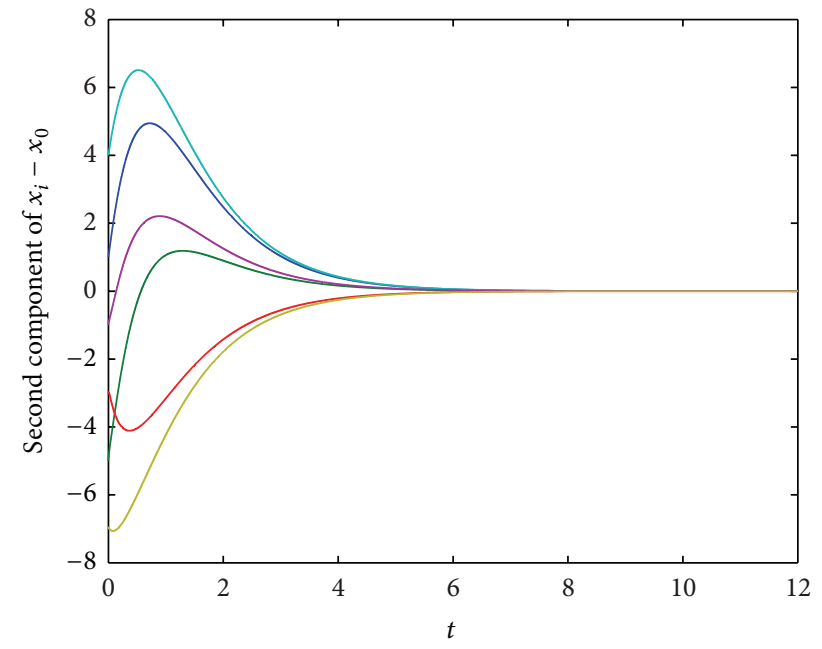

(b)

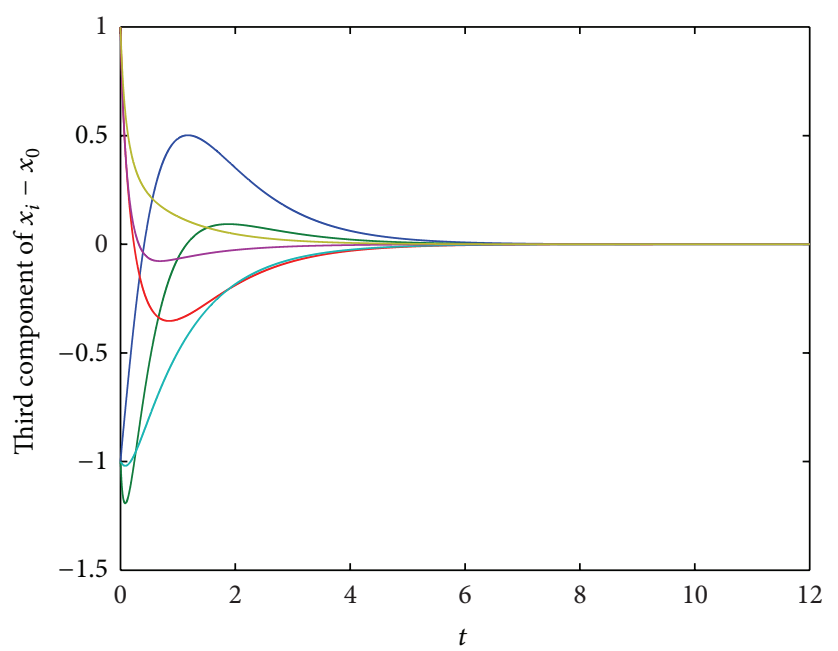

(c)

FIGURE 3: The error trajectories between the leader and each agent.

Take $Q=I$ and then solve the Riccati equation (11) to get the unique positive define solution $P$. Thus, the gain matrix $L$ can be constructed by

$$
L=P C^{T}=\left(\begin{array}{cc}
3.4019 & 0.7514 \\
-2.0167 & -0.0430 \\
-4.0747 & -1.3478
\end{array}\right) .
$$

Matrix $A$ is not stable. Select feedback matrix

$$
K=\left(\begin{array}{ccc}
-1.6429 & 0.2143 & 1.2143 \\
0.2143 & -0.0714 & -1.0714
\end{array}\right)
$$

such that $A-B K$ is stable.

The initial state of all agents is randomly produced. The state errors showed in Figures 2 and 3 are $x_{i 1}-x_{01}, x_{i 2}-x_{02}$ and $x_{i 3}-x_{03}$, respectively.

We first use the approach proposed in Section 3 to solve the consensus problem; that is, each agent uses the feedback control law (10) together with the distributed estimation law
(9). The trajectories of $x_{i j}-x_{0 j}, j=1,2,3$, are depicted in Figure 2, which shows that the follower agents can track the leader agent.

Next, we use the feedback control law (10) together with the distributed estimation law (9) for agent $i$ to solve the consensus problem. The trajectories of $x_{i j}-x_{0 j}, j=1,2,3$, are depicted in Figure 3, which also shows that the followeragents can track the leader agent.

\section{Conclusions}

In this paper, the leader-following consensus problem for multi-agent systems with general form of linear dynamics and undirected switching topologies has been investigated. Based on the relative outputs of neighboring agents, a distributed observer-based consensus protocol is proposed to each following agent to track the leader. A multialgorithm has been proposed to construct the consensus protocol, and the control gain matrices used in the consensus protocol are 
obtained by solving the Riccati equation and the Sylvester equation. A sufficient consensus condition is established by using parameter-dependent lyapunov function method under switching topologies. By using the analysis method of this paper, it is easy to establish the distributed observerbased consensus protocol for multi-agent systems under leaderless case. We also will probe multi-agent robust consensus control problems with external disturbance under time-delay switching topologies in our future work.

\section{Acknowledgments}

This work was supported by the National natural Science Foundation of China under Grants 61074123 and 61174063 and the open project of State Key Laboratory of Industrial Control Technology in Zhejiang University, China, under Grant no. ICT1218.

\section{References}

[1] R. M. Murray, "Recent research in cooperative control of multivehicle systems," Journal of Dynamic Systems, Measurement and Control, Transactions of the ASME, vol. 129, no. 5, pp. 571-583, 2007.

[2] W. Ren and R. W. Beard, Distributed Consensus in Multi-Vehicle Cooperative Control: Theory and Applications, Springer, Berlin, Germany, 2008.

[3] A. Jadbabaie, J. Lin, and A. S. Morse, "Coordination of groups of mobile autonomous agents using nearest neighbor rules," IEEE Transactions on Automatic Control, vol. 48, no. 6, pp. 988-1001, 2003.

[4] W. Ren and R. W. Beard, "Consensus seeking in multiagent systems under dynamically changing interaction topologies," IEEE Transactions on Automatic Control, vol. 50, no. 5, pp. 655661, 2005.

[5] L. Gao, D. Cheng, and Y. Hong, "Control of group of mobile autonomous agents via local strategies," Journal of Control Theory and Applications, vol. 6, no. 4, pp. 357-364, 2008.

[6] Z.-H. Guan, Y. Wu, and G. Feng, "Consensus analysis based on impulsive systems in multiagent networks," IEEE Transactions on Circuits and Systems. I. Regular Papers, vol. 59, no. 1, pp. 170178, 2012.

[7] R. Olfati-Saber and R. M. Murray, "Consensus problems in networks of agents with switching topology and time-delays," IEEE Transactions on Automatic Control, vol. 49, no. 9, pp. 15201533, 2004.

[8] Z. Lin, M. Broucke, and B. Francis, "Local control strategies for groups of mobile autonomous agents," IEEE Transactions on Automatic Control, vol. 49, no. 4, pp. 622-629, 2004.

[9] Y. Cao and W. Ren, "Sampled-data discrete-time coordination algorithms for double-integrator dynamics under dynamic directed interaction," International Journal of Control, vol. 83, no. 3, pp. 506-515, 2010.

[10] Y. Hong, J. Hu, and L. Gao, "Tracking control for multiagent consensus with an active leader and variable topology," Automatica, vol. 42, no. 7, pp. 1177-1182, 2006.

[11] J. Hu and Y. Hong, "Leader-following coordination of multiagent systems with coupling time delays," Physica A, vol. 374, no. 2, pp. 853-863, 2007.
[12] Y. Hong, G. Chen, and L. Bushnell, "Distributed observers design for leader-following control of multi-agent networks," Automatica, vol. 44, no. 3, pp. 846-850, 2008.

[13] L. Gao, J. Zhang, and W. Chen, "Second-order consensus for multiagent systems under directed and switching topologies," Mathematical Problems in Engineering, vol. 2012, Article ID 273140, 21 pages, 2012.

[14] F. Xiao and L. Wang, "Consensus problems for high-dimensional multi-agent systems," IET Control Theory and Applications, vol. 1, no. 3, pp. 830-837, 2007.

[15] W. Yu, G. Chen, and M. Cao, "Some necessary and sufficient conditions for second-order consensus in multi-agent dynamical systems," Automatica, vol. 46, no. 6, pp. 1089-1095, 2010.

[16] K. Peng and Y. Yang, "Leader-following consensus problem with a varying-velocity leader and time-varying delays," Physica A, vol. 388, no. 2-3, pp. 193-208, 2009.

[17] L. Gao, X. Zhu, and W. Chen, "Leader-following consensus problem with an accelerated motion leader," International Journal of Control, Automation, and Systems, vol. 10, no. 5, pp. 931-939, 2012.

[18] V. Ugrinovskii, "Distributed robust filtering with $H_{\infty}$ consensus of estimates," Automatica, vol. 47, no. 1, pp. 1-13, 2011.

[19] Y. Tang, H. Gao, W. Zou, and J. Kurths, "Distributed synchronization in networks of agent systems with nonlinearities and random switchings," IEEE Transactions on Systems, Man, and Cybernetics, Part B, vol. 43, pp. 358-370, 2013.

[20] Y. Tang and W. K. Wong, "Distributed synchronization of coupled neural networks via randomly occurring control," IEEE Transactions on Neural Networks and Learning Systems, vol. 24, no. 3, pp. 435-447, 2013.

[21] D. Jin and L. Gao, "Stability analysis of a double integrator swarm model related to position and velocity," Transactions of the Institute of Measurement and Control, vol. 30, no. 3-4, pp. 275-293, 2008.

[22] N. Cai, J.-X. Xi, and Y.-S. Zhong, "Swarm stability of high-order linear time-invariant swarm systems," IET Control Theory \& Applications, vol. 5, no. 2, pp. 402-408, 2011.

[23] W. Zhang, Y. Tang, J. Fang, and X. Wu, "Stability of delayed neural networks with time-varying impulses," Neural Networks, vol. 36, pp. 59-63, 2012.

[24] L. Gao, Y. Tang, W. Chen, and H. Zhang, "Consensus seeking in multi-agent systems with an active leader and communication delays," Kybernetika, vol. 47, no. 5, pp. 773-789, 2011.

[25] S. Li, H. Du, and X. Lin, "Finite-time consensus algorithm for multi-agent systems with double-integrator dynamics," Automatica, vol. 47, no. 8, pp. 1706-1712, 2011.

[26] G. Wen, Z. Duan, W. Yu, and G. Chen, "Consensus in multiagent systems with communication constraints," International Journal of Robust and Nonlinear Control, vol. 22, no. 2, pp. 170 $182,2012$.

[27] D. Hammel, "Formation flight as an energy saving mechanism," Israel Journal of Zool, vol. 41, pp. 261-278, 1995.

[28] M. Andersson and J. Wallander, "Kin selection and reciprocity in flight formation," Behavioral Ecology, vol. 15, no. 1, pp. 158162, 2004.

[29] B. Liu, T. Chu, L. Wang, and G. Xie, "Controllability of a leader-follower dynamic network with switching topology," IEEE Transactions on Automatic Control, vol. 53, no. 4, pp. 10091013, 2008.

[30] W. Ni and D. Cheng, "Leader-following consensus of multiagent systems under fixed and switching topologies," Systems \& Control Letters, vol. 59, no. 3-4, pp. 209-217, 2010. 
[31] E. Tian, D. Yue, and C. Peng, "Quantized output feedback control for networked control systems," Information Sciences, vol. 178 , no. 12 , pp. $2734-2749,2008$.

[32] A. Abdessameud and A. Tayebi, "On consensus algorithms for double-integrator dynamics without velocity measurements and with input constraints," Systems \& Control Letters, vol. 59, no. 12, pp. 812-821, 2010.

[33] W. Dong, "Distributed observer-based cooperative control of multiple nonholonomic mobile agents," International Journal of Systems Science, vol. 43, no. 5, pp. 797-808, 2012.

[34] Y. Hong and X. Wang, "Multi-agent tracking of a high-dimensional active leader with switching topology," Journal of Systems Science \& Complexity, vol. 22, no. 4, pp. 722-731, 2009.

[35] J. H. Seo, H. Shim, and J. Back, "Consensus of high-order linear systems using dynamic output feedback compensator: low gain approach," Automatica, vol. 45, no. 11, pp. 2659-2664, 2009.

[36] Z. Li, Z. Duan, and G. Chen, "Dynamic consensus of linear multi-agent systems," IET Control Theory \& Applications, vol. 5, no. 1, pp. 19-28, 2011.

[37] Z. Li, Z. Duan, G. Chen, and L. Huang, "Consensus of multiagent systems and synchronization of complex networks: a unified viewpoint," IEEE Transactions on Circuits and Systems. I. Regular Papers, vol. 57, no. 1, pp. 213-224, 2010.

[38] G. Wen, Z. Li, Z. Duan, and G. Chen, "Distributed consensus control for linear multi-agent systems with discontinuous observations," International Journal of Control, vol. 86, no. 1, pp. 95-106, 2013.

[39] C. Godsil and G. Royle, Algebraic Graph Theory, vol. 207, Springer, New York, NY, USA, 2001.

[40] R. A. Horn and C. R. Johnson, Matrix Analysis, Cambridge University Press, New York, NY, USA, 1985.

[41] W. M. Wonham, Linear Multivariable Contol, vol. 10, Springer, New York, NY, USA, 1985.

[42] C. T. Chen, Linear System Theory and Design, Oxford University Press, New York, NY, USA, 3rd edition, 1999. 


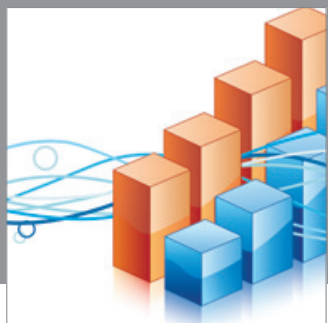

Advances in

Operations Research

mansans

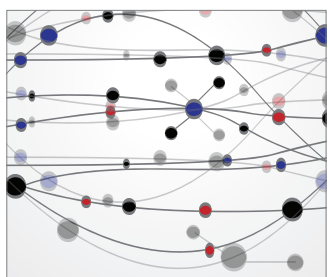

The Scientific World Journal
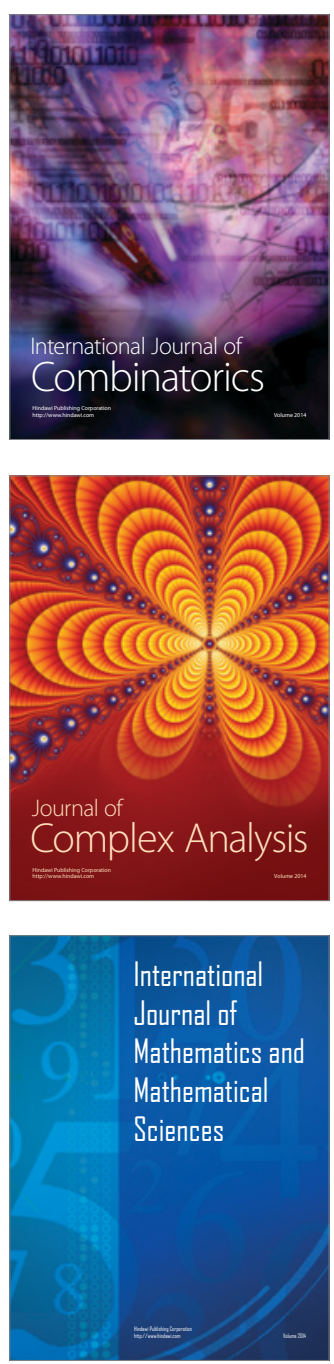
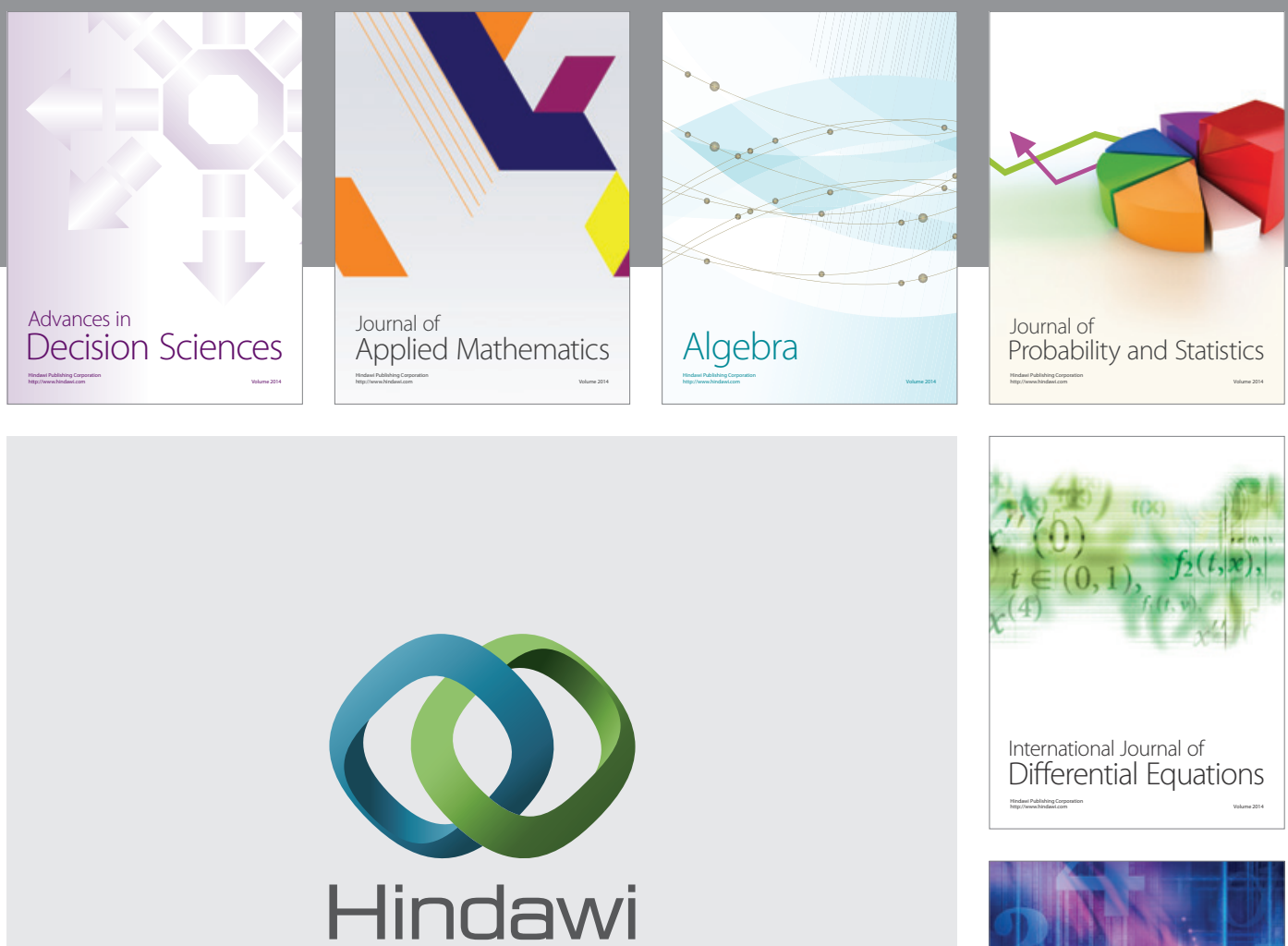

Submit your manuscripts at http://www.hindawi.com
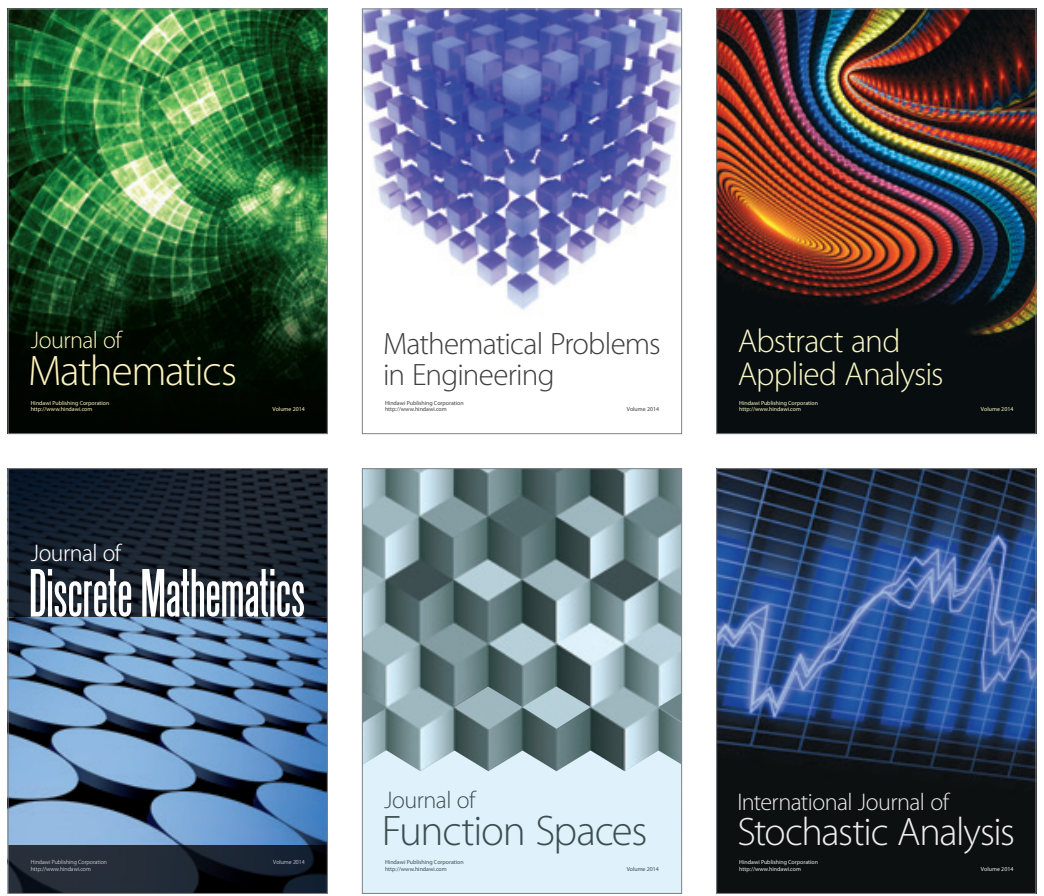

Journal of

Function Spaces

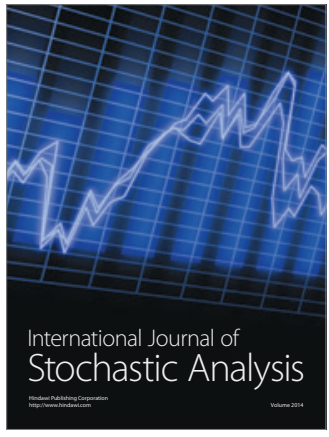

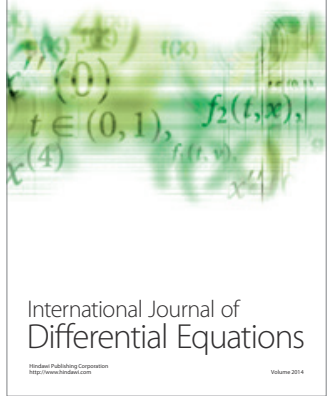
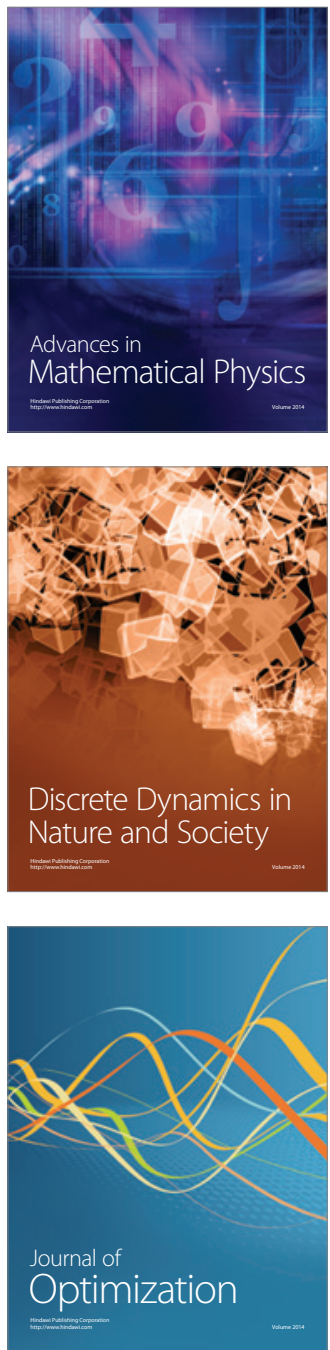